\title{
Liquid Pricing for Digital Infrastructure Services
}

\author{
Subhajyoti Bandyopadhyay* \\ and \\ Hsing Kenneth Cheng \\ Department of Decision and Information Sciences \\ Warrington College of Business Administration \\ University of Florida \\ Gainesville, FL 32611-7169
}

* Corresponding author. Phone (352) 392-5946 Fax (352) 392-5438 e-mail: shubho.bandyopadhyay@cba.ufl.edu Authors are in alphabetical order. 


\title{
Liquid Pricing For Digital Infrastructure Services
}

\begin{abstract}
Broadband access providers today have an overcapacity in infrastructure, while their service penetration remains low. This has led to the introduction of intermediate service levels, so that first-time consumers start experiencing the benefits of these new services. We analyze the effect of a novel pricing scheme, called 'liquid bandwidth', whereby customers can upgrade their services for a small period of time when they want to utilize broadband services for newer types of online content, by paying per-unit access charges. The results indicate that introduction of such pricing schemes can significantly boost the profits of the service provider. Further, effective penetration of broadband services in a monopolist market might require regulatory intervention or some form of subsidy.
\end{abstract}

Keywords: liquid pricing; broadband access pricing; digital infrastructure services; market segmentation; universal access; public policy; market regulation 


\section{Introduction}

The large-scale deployment of broadband services has brought about a glut in the infrastructure capacity of the service providers. While $94 \%$ of the zip codes in the United States have the capability for broadband, the number of broadband users stood at a relatively modest 28.2 million in December $2003^{1}$ [2]. In terms of broadband penetration among its population, the United States is currently ranked eleventh in the world. In the arena of wireless services, the providers are expected to start offering services like wireless Internet at broadband speeds, but their adoption remains an open question [18].

In a bid to utilize the available infrastructure, broadband service providers are thus testing new strategies to attract new customers. In the United Sates, given the low penetration of broadband services and the widespread infrastructure overcapacity, service providers have started offering 'lite' or intermediate versions of their offerings at lower prices. ${ }^{2}$ The underlying strategy is to gain a foothold into the households of hitherto dialup customers, who can presumably be later convinced to upgrade to higher broadband speeds, after they experience the benefits of these services. Further, the rise of digital services like music downloads, video streaming and even full-length feature film downloads might even lead to the rise of new segment of consumers who might not be heavy users of the "traditional” internet, but who might value these new services much more. Many cable and DSL Internet service providers, therefore, now regularly offer download speed offerings typically in the range of 200-300 kbps, at monthly prices that are lower than

\footnotetext{
${ }^{1}$ The FCC defines any speed exceeding 200 kbps as a broadband connection

${ }^{2}$ In the authors' neighborhood, for example, the cable broadband service provider introduced an 'introductory' 256kbps download speed, as compared to the existing 4Mbps and 5Mbps download speeds (i.e. roughly 15 and 19 times higher speeds). A similar offering structure exists for the local DSL broadband providers.
} 
their standard offerings. In short, a confluence of several factors have led the broadband services providers to experiment with new pricing models that differ significantly from the flat-rate pricing that has been common from the early days of commercial internet access.

A related issue is the very availability of broadband services (this is different issue from capability for broadband, as a service provider might choose not to roll out broadband services in an area that with limited demand for such services even though the basic telecommunications infrastructure might be in place). Access to online information through the internet has now entered public policy debates. Several broadband service providers have been charged (with one class-action lawsuit in Florida against AT\&T Broadband) with rolling out their services only in high-income areas, and a high-ranking member of the U.S. House telecommunications subcommittee has expressed concern with these developments [9]. Some analysts argue that there is now need for government intervention to ensure universal broadband access [10], one idea being that there is a subsidy akin to the Universal Service Funds tax that telecommunications companies today charge their subscribers to subsidize increased telephone access to schools and rural areas [21].

We analyze the effect of various internet pricing strategies with this backdrop. The basic driver of this analysis is a promising and innovative service, already available in some Scandinavian countries, that allows DSL subscribers to boost their connection speeds for a limited period for a small fee, a concept that has been dubbed "liquid bandwidth" [1]. 
The underlying idea is that some consumers might sometimes want to download a large file (for example, a new software, a song or a media clip, or even a full-length feature film), and would prefer the higher speeds for those limited timeframes. These users would prefer to pay lower costs for the service for their typical usage (like checking email, online buying, or regular web surfing), but would find that service too limited for downloading large files. Currently, the service levels provided by the service providers fail to catch this opportunity for these classes of users, who either do not sufficiently utilize the high-bandwidth content, or do not use them at all after considering the agonizing wait at their service level. With the rapid growth of online content (in the form of downloadable songs, real-time entertainment and gradually full-length feature films), and the overwhelmingly large population of users still using narrowband access, the size of this missed opportunity gets larger by the day. ${ }^{3}$ These users might not value their traditional Internet access very highly, but would be willing to pay more for the new online digital content. In the corporate segment, liquid bandwidth is already a functional concept in some places - a company in the United Kingdom, Neos Networks ${ }^{4}$, provides upgrades to corporate customers that range from $30 \mathrm{MB} / \mathrm{s}$ to $100 \mathrm{MB} / \mathrm{s}$ within ten minutes.

[4] argues that the lack of segmentation in broadband services is a direct result of the fact that service providers do not offer quality of service guarantees, and therefore customers do not value different levels of services. They point to the lack of empirical evidence of customers paying more for relatively better performances. In recent times, however,

\footnotetext{
${ }^{3}$ In fact, the content service providers have a possible revenue-sharing opportunity with the providers of these nascent high-bandwidth services.

${ }^{4}$ http://www.neosnetworks.net/
} 
several broadband access providers have started to introduce different levels of service, and anecdotal evidence seems to suggest that consumers are gradually differentiating between different classes of service insofar as their service offerings are sufficiently different, and are willing to pay different prices for them depending on the delay they incur while waiting for their service. ${ }^{5}$ In this paper, we therefore model the consumers' choice emanating directly from their different levels of disutility of waiting for the service. In other words, consumers who would not mind the congestion created by others would have a lower delay cost as compared to consumers who do not like waiting for their Internet download to take place, regardless of the type of usage.

This paper looks at the various models of market segmentation in broadband internet access, and analyzes the viability of the new pricing strategies. In particular, we try to analyze whether the concept of 'liquid pricing' of broadband access services increases the profits of the service providers. These pricing strategies are in no way limited to just broadband internet access, and can be in fact modified for pricing of digital infrastructure services in general. It also considers the public policy implications in such pricing strategies - e.g. under what market conditions would there be a need for subsidies in order to ensure market coverage [3]?

The INDEX project trial at the University of California at Berkeley [7] demonstrated clearly that flat-rate pricing of internet service providers (or ISPs) is very inefficient and hinders deployment of broadband access. Further, it showed that quality-differentiated

\footnotetext{
${ }^{5}$ For example, other than the example in Footnote 2, several DSL service providers, like BellSouth, SBC or EarthLink, regularly offer three levels of broadband services differentiated by the download speeds - 256 kbps, 1.5 Mbps and 3.0 Mbps.
} 
service levels provide higher levels of surplus for both the consumers and service providers. Market segmentation based on consumers’ utility levels and willingness to pay is a well-known concept. While providing different download speeds for a fixed fee is definitely a more efficient strategy than 'one price fits all', the concept of 'liquid bandwidth' provides the opportunity of better customer segmentation and the ability to cater to customers who value traditional static "informational” online access differently from the newer online content. A consumer today can call her service provider and immediately get upgraded to a higher bandwidth, and the entire process is controlled through software. ${ }^{6}$ Thus, from a technological point of view, the problem is relatively simple, and the marginal cost of implementation - especially at current levels of infrastructure utilization - is very close to zero. What is required is a simple billing interface that the consumers can interact with - either by calling the customer service or by ordering from the Internet (the latter allowing further lowering of the transaction costs).

Our paper differs from the current literature in the way it explicitly allows for modeling the above-mentioned opportunity. We model the Internet traffic and its pricing in terms of congestion costs for various types of users. We consider the pricing for Internet access on a per-unit basis (the unit being of course time), and find conditions for equilibrium under different scenarios of service offerings and consumer types.

\section{Literature review}

\footnotetext{
${ }^{6}$ One of the authors got his service upgraded to 5.0 Mbps, tested the download speed, and then downgraded the service to $4.0 \mathrm{Mbps}$, tested it again, all within the duration of one phone call.
} 
Market segmentation is an effective tool to extract consumer surplus when there is sufficient heterogeneity among customers, see for example, [6, 13, 17, 19, 22].

Several existing literature on tiered pricing for Internet services focus on the quality of service at the level of an individual packet. For example, [14] and [15] propose a "smart market” for Internet pricing, whereby the individual packets contain a bid value that is set by the originator of the packet, and packets with higher bid values are given priority to clear the (congested) network. [20] proposes a "Paris Metro Pricing” framework, where the network consists of several subnetworks that are identical in terms of operation, but differ in price. Users therefore self-select the networks based on the valuation of their packets and expected congestion. Several papers address the main architectural criticism of this framework, namely its inability to provide quality of service (QoS) guarantees, and a summary of that literature can be found in [4].

[4] proposes a novel quality-contingent pricing scheme and considers two mechanisms a proportional rebate based on number of packet losses or full rebate if the quality falls below a certain threshold (see also [5] for a detailed discussion on the full-price rebate model). [4] assumes that users in a community receive the same level of service, and therefore suggest differentiating between them on the level of service guarantee. They therefore suggest a monitoring equipment for recording QoS between the service provider and the end-users that is maintained by an independent auditing body. They then go on to suggest the two aforementioned pricing schemes. The model therefore provides an interesting and novel variation on access pricing in terms of quality of services 
guarantees. However, it is based on the premise that all users receive the same class of service, and therefore cannot be applicable when the provider specifically introduces multiple classes of service. In contrast, our model specifically assumes that the service provider introduces different classes of service that caters to different types of user needs.

Our model extends the congestion pricing model suggested by [16], whereby a pricing model is proposed by taking into consideration the value of the users' time. It also has stylistic similarities to the modeling of [11]. We assume that there are different classes of users, who value their cost of delay (in terms of dollars per unit time) in a congested network differently, which in turn provides for the opportunity to segment the consumers. The model takes cognizance of the fact that currently there are different classes of service, and different classes of users who value their access delays differently. It also takes into account the fact that there are new types of digital content that might make current pricing implementations limiting for a large number of users. Finally, since the technology for implementing its pricing scheme already exists, there is no need for any added hardware to implement it.

\section{Model assumptions}

For simplification purposes, we assume that there are two levels of consumer demand, denoted by $H$ and $L$, although there can be more than two service offerings. In other words, if the service in question were a broadband Internet service, there would be two possible classes of consumers. Consumers who would want the higher speed regardless of usage are classified the high-type $(H)$, and the consumers who would regularly want 
the lower speed are classified the low-type $(L)$. In our model, the service provider is assumed to be exclusively a high-bandwidth provider. Thus, other than the decision regarding introduction of liquid pricing of on-demand services, the provider has to decide whether to cater to just the high-type customers or both types of customers. We assume that most of the consumers are of the latter type, i.e. the consumers who want speedy access at all times are relatively small in number. We also assume that the low-type consumers feel the need for high-bandwidth services for a relatively small fraction of the time. We will later specify in greater detail what these relatively small quantities mean in the context of the model. We assume a fixed size of a packet regardless of the type of usage, and the usage levels differ only in the arrival rates of the packets, which is a reasonable assumption for Internet traffic. The two types of customers are differentiated in terms of the arrival rates of these packets: the high-type customers have a much higher arrival rate of these packets as compared to the low-type customers. We assume that the service provider has different fixed fees per unit of time for the two levels of services, and a per-unit charge (i.e. liquid pricing) for on-demand access. Finally, for tractability purposes, we make the simplifying assumption that the two types of users have the entire channel bandwidth dedicated to themselves when they are using a particular channel. ${ }^{7}$

\section{The Model}

We consider two scenarios for our model in order to distinguish the effect of offering the low-type users to utilize bandwidth on demand. In both scenarios, we make the same assumptions regarding the service offerings and the two types of consumers. The first

\footnotetext{
${ }^{7}$ In terms of the two most common forms of broadband internet access today (cable and DSL), it needs to be clarified that we model the congestion at the 'back end' of the service provider (between the provider and the Internet). We thank one anonymous reviewer for suggesting this clarification.
} 
scenario discusses the model that is more prevalent until now - a broadband provider who provides a single level of service, regardless of the user type, just like the assumption of [4]. In the second scenario, the broadband service provider has two classes of service, and has the option of providing liquid pricing for on-demand access to the low-type customers as and when they request it.

\section{Model 1}

We apply a M/M/1 queuing system to describe the service system where the high-type customers are characterized by a Poisson arrival rate of requests (packets) per unit of time $\lambda_{H}$, while the low-type customer's Poisson arrival rate of requests (packets) per unit of time is $\lambda_{L}$ and $\lambda_{H} \gg \lambda_{L}$. The service provider has the capacity of processing $\mu$ requests per unit time. Let $N_{H}$ and $N_{L}$ represent the numbers of high type and low type customers respectively. Further, let $d_{H}$ be the delay cost per unit of time of high-type customers and the delay cost per unit of time of low-type customers is $d_{L}$. Naturally, $d_{H}>d_{L}$. The gross value function of using the service for the two types of customers is described by $v\left(\lambda_{k}\right), k \in\{H, L\}$. The $v\left(\lambda_{k}\right)$ 's are twice differentiable and concave, and $v\left(\lambda_{H}\right) \gg v\left(\lambda_{L}\right)$

Thus, in this scenario, we assume that the service provider has one channel of service to serve all her customers. The capacity is enough to serve $\mu$ service requests (packets) per unit of time. As is to be expected, the high-type customers place a premium on their service, and therefore their disutility of waiting is higher $\left(d_{H}>d_{L}\right)$. Further, since the 
valuation of a service is likely to increase with the number of packets sent, and the arrival rate of packets is significantly higher at broadband speeds, we assume that $v\left(\lambda_{H}\right) \gg v\left(\lambda_{L}\right)$

\section{Analysis}

A provider who has the technology to provide high bandwidth services can decide on whether to sell to the high-type customers only or cater to both types. She can also decide whether she wants to introduce liquid pricing, or go for only fixed fee pricing. These strategies are summarized in Table 1:

Table 1: Product Strategies of the Digital Infrastructure Services

\begin{tabular}{lll}
\hline & Sell to High type only & Sell to both types \\
\hline No on-demand & Option 1 & Option 2 \\
& $\Pi_{1}=N_{H} F_{1}$ & $\Pi_{2}=\left(N_{H}+N_{L}\right) F_{2}$ \\
\hline Introduce on-demand & Option 3 & Option 4 \\
& $\Pi_{3}=N_{H} p_{1} \lambda_{H}$ & $\Pi_{4}=N_{H} F_{3}+N_{L} p_{2} \lambda_{L}$ \\
\hline
\end{tabular}

The $F_{i}, i \in\{1,2,3\}$, in Table 1 refers to the various fixed prices in Options 1,2 and 4 respectively, while $p_{j}, j \in\{1,2\}$, represents the liquid pricing, i.e. usage charge per unit of time in Options 3 and $4 .^{8}$ It needs to be clarified that the two periods of time for

\footnotetext{
${ }^{8}$ Since the provider has just one level of service in this model, the implicit assumption in option 4 is that the low-type customer utilizes its services only during the times he needs broadband access, and therefore has some other provider for its "usual" internet access requirements. The setting of model 1 is somewhat artificial that way, since there is only one channel. In this setting, if the low-type user is given a (presumably lower) fixed access charge for his usual needs, he will have no need to pay per unit access charges for his broadband needs (since he gets to use that same channel through his fixed fee). Further, the high-type user would have no incentive to pay a higher fixed cost for the same service, and thus Option 4 would reduce to Option 2. This artificiality is taken care of in Model 2, which expectedly gets more
} 
charging the fixed price and the liquid pricing are different - the fixed pricing can be thought of as the monthly charges that is common today, while the unit time for the liquid pricing would probably be a slot of a few hours.

The profit maximization principle of the provider in each of the above options is as follows:

Option 1:

$$
\begin{aligned}
& \max _{F_{1}} \Pi_{1}=N_{H} F_{1} \\
& \text { s.t. } \quad v\left(\lambda_{H}\right)-\frac{d_{H}}{\mu-N_{H} \lambda_{H}}-F_{1} \geq 0
\end{aligned}
$$

\section{Option 2:}

$$
\begin{aligned}
& \max _{F_{2}} \Pi_{2}=\left(N_{H}+N_{L}\right) F_{2} \\
& \text { s.t. } \\
& v\left(\lambda_{L}\right)-\frac{d_{L}}{\mu-\left(N_{H} \lambda_{H}+N_{L} \lambda_{L}\right)}-F_{2} \geq 0 \\
& v\left(\lambda_{H}\right)-\frac{d_{H}}{\mu-\left(N_{H} \lambda_{H}+N_{L} \lambda_{L}\right)}-F_{2} \geq 0
\end{aligned}
$$

\section{Option 3:}

involved. Similarly, the high-type user will have no need to pay on-demand access charges along with a fixed fee in Option 3, since he already has access to the same channel (and its lower congestion) though Option 1, and therefore Option 3 will reduce to Option 1. Our idea was to introduce first a simplified Model 1 to give a general flavor of the problem and its implications, and then follow it up with the more realistic Model 2. 


$$
\begin{aligned}
& \max _{p_{1}} \Pi_{3}=N_{H} p_{1} \lambda_{H} \\
& \text { s.t. } \quad v\left(\lambda_{H}\right)-\frac{d_{H}}{\mu-N_{H} \lambda_{H}}-p_{1} \lambda_{H} \geq 0
\end{aligned}
$$

\section{Option 4:}

$$
\max _{F_{3}, p_{2}} \Pi_{4}=N_{H} F_{3}+N_{L} p_{2} \lambda_{L}
$$

s.t.

$$
\begin{gathered}
v\left(\lambda_{L}\right)-\frac{d_{L}}{\mu-\left(N_{H} \lambda_{H}+N_{L} \lambda_{L}\right)}-p_{2} \lambda_{L} \geq v\left(\lambda_{L}\right)-\frac{d_{L}}{\mu-\left(N_{H} \lambda_{H}+N_{L} \lambda_{L}\right)}-F_{3} \\
v\left(\lambda_{H}\right)-\frac{d_{H}}{\mu-\left(N_{H} \lambda_{H}+N_{L} \lambda_{L}\right)}-F_{3} \geq v\left(\lambda_{H}\right)-\frac{d_{H}}{\mu-\left(N_{H} \lambda_{H}+N_{L} \lambda_{L}\right)}-p_{2} \lambda_{H} \\
v\left(\lambda_{L}\right)-\frac{d_{L}}{\mu-\left(N_{H} \lambda_{H}+N_{L} \lambda_{L}\right)}-p_{2} \lambda_{L} \geq 0 \\
v\left(\lambda_{H}\right)-\frac{d_{H}}{\mu-\left(N_{H} \lambda_{H}+N_{L} \lambda_{L}\right)}-F_{3} \geq 0
\end{gathered}
$$

In option 1, the single constraint (1) is binding, as the provider will raise the price of the service such that it is just worthwhile for the high-type consumers to participate. Similarly, in option 3, the single condition is binding. Thus, we get in option 1 ,

$$
F_{1}=v\left(\lambda_{H}\right)-\frac{d_{H}}{\mu-N_{H} \lambda_{H}}
$$

And in option 3,

$$
p_{1}=\frac{v\left(\lambda_{H}\right)}{\lambda_{H}}-\frac{d_{H} / \lambda_{H}}{\mu-N_{H} \lambda_{H}}
$$

The profit to the provider is the same in both cases, so that 


$$
\Pi_{1}=\Pi_{3}=N_{H}\left(v\left(\lambda_{H}\right)-\frac{d_{H}}{\mu-N_{H} \lambda_{H}}\right)
$$

Given the fact that option 1 and 3 yield the same profit, we comment that in practical terms, the service provider would probably introduce just option 1 , given the possible complexities of billing for liquid pricing, especially when it does not yield any extra profit. We also note that the consumer surplus in both these two options is zero, as the single conditions are binding.

In option 2, the two constraints are essentially the participation constraints of the lowand high-type users respectively. Since $v\left(\lambda_{H}\right) \gg v\left(\lambda_{L}\right)$, constraint (3) becomes strictly positive, and therefore the high-type consumer has a strictly positive surplus. The service provider raises the price of the service so that it is just worthwhile for the low-type to participate for the service, so that the first constraint becomes binding (the low-type consumer thus has zero surplus). Therefore, $v\left(\lambda_{L}\right)-\frac{d_{L}}{\mu-\left(N_{H} \lambda_{H}+N_{L} \lambda_{L}\right)}-F_{2}=0$, or

$$
F_{2}=v\left(\lambda_{L}\right)-\frac{d_{L}}{\mu-\left(N_{H} \lambda_{H}+N_{L} \lambda_{L}\right)}
$$

and

$$
\Pi_{2}=\left(N_{H}+N_{L}\right)\left(v\left(\lambda_{L}\right)-\frac{d_{L}}{\mu-\left(N_{H} \lambda_{H}+N_{L} \lambda_{L}\right)}\right)
$$

We note that the consumer surplus of the high-type consumer is given by the expression

$$
v\left(\lambda_{H}\right)-v\left(\lambda_{L}\right)-\left(\frac{d_{H}}{\mu-\left(N_{H} \lambda_{H}+N_{L} \lambda_{L}\right)}-\frac{d_{L}}{\mu-\left(N_{H} \lambda_{H}+N_{L} \lambda_{L}\right)}\right) .
$$


Option 4 gives us the standard formulation of the mechanism design of the two-menu, two-customer model, where (5) and (6) are the incentive compatibility constraints and (7) and (8) are the participation constraints of the low- and high-type users respectively. It is common for service providers to price their services higher when they charge on a perunit basis, as compared to when they charge a fixed fee, especially at higher usage levels. ${ }^{9}$ Following this common practice, the service provider in our model will increase the (fixed) price of the high-type user to such an extent that he finds it just worth his while to pay the fixed price. In other words, constraint (6) will be binding. Thus,

$$
v\left(\lambda_{H}\right)-\frac{d_{H}}{\mu-\left(N_{H} \lambda_{H}+N_{L} \lambda_{L}\right)}-F_{3}=v\left(\lambda_{H}\right)-\frac{d_{H}}{\mu-\left(N_{H} \lambda_{H}+N_{L} \lambda_{L}\right)}-p_{2} \lambda_{H} .
$$

This leads to $F_{3}=p_{2} \lambda_{H}$, and substituting this value of $F_{3}$ in (5) shows that constraint (5) will not be binding. Also, since $v\left(\lambda_{H}\right) \gg v\left(\lambda_{L}\right)$, we can assume that the net utility of the of high-type user is higher than that of the low-type user, i.e.

$$
v\left(\lambda_{H}\right)-\frac{d_{H}}{\mu-\left(N_{H} \lambda_{H}+N_{L} \lambda_{L}\right)}-F_{3} \geq v\left(\lambda_{L}\right)-\frac{d_{L}}{\mu-\left(N_{H} \lambda_{H}+N_{L} \lambda_{L}\right)}-p_{2} \lambda_{L} .
$$

Since the right-hand side of the above inequality is the net utility of the low-type user, his participation constraint ensures that the participation constraint of the high-type user is not binding. The service provider increases the liquid price of the low-type user so that he just participates. Therefore,

$$
v\left(\lambda_{L}\right)-\frac{d_{L}}{\mu-\left(N_{H} \lambda_{H}+N_{L} \lambda_{L}\right)}-p_{2} \lambda_{L}=0 .
$$

\footnotetext{
${ }^{9}$ For example, cell phone service providers charge higher for the so-called pre-paid options (where the subscribers pay by the minute) than for services that have a fixed service cost per month. The same phenomenon is observed in broadband Internet access services - in the authors' local area for example, the cable broadband connection service offers a 256kbps download service at approximately \$25 per month, and $4 \mathrm{Mbps}$ and $5 \mathrm{Mbps}$ download services at $\$ 40$ and $\$ 55$ per month respectively (the upload speeds also vary considerably for these services).
} 
Thus,

$$
F_{3}=p_{2} \lambda_{H}
$$

and

$$
p_{2}=\frac{v\left(\lambda_{L}\right)}{\lambda_{L}}-\frac{d_{L} / \lambda_{L}}{\mu-\left(N_{H} \lambda_{H}+N_{L} \lambda_{L}\right)}
$$

Therefore, the total profits of the provider in option 4 is given by

$$
\Pi_{4}=p_{2}\left(N_{H} \lambda_{H}+N_{L} \lambda_{L}\right)=\left(\frac{v\left(\lambda_{L}\right)}{\lambda_{L}}-\frac{d_{L} / \lambda_{L}}{\mu-\left(N_{H} \lambda_{H}+N_{L} \lambda_{L}\right)}\right)\left(N_{H} \lambda_{H}+N_{L} \lambda_{L}\right)
$$

As is to be expected, given the binding participation constraint, this profit depends on the delay cost of the low-type customer, and not that of the high-type customer. Further, as expected, the low-type consumer does not have any surplus, while the high-type consumer has a strictly positive surplus, given by $v\left(\lambda_{H}\right)-\frac{d_{H}}{\mu-\left(N_{H} \lambda_{H}+N_{L} \lambda_{L}\right)}-\lambda_{H}\left(\frac{v\left(\lambda_{L}\right)}{\lambda_{L}}-\frac{d_{L} / \lambda_{L}}{\mu-\left(N_{H} \lambda_{H}+N_{L} \lambda_{L}\right)}\right)$.

Depending on the functional form of the gross value function $v(\lambda)$, the relative magnitudes of the two types of customers, and their delay costs, the provider will rank the various profit functions, and choose the strategy that gives her the highest profits. Given the large number of parameters, and the functional form of the equilibrium, it is difficult to make an analytical comparison between the various expressions of profit, so instead we consider a numerical example. 
Following [16], we assume an isoelastic demand function, such that $v^{\prime}\left(\lambda_{k}\right)=k / \lambda^{a}$, so that $v\left(\lambda_{k}\right)=\frac{k \lambda^{1-a}}{1-a}$. In our stylized framework, the absolute values of the parameters are not so important as the relative magnitudes of the parameters of the two different types of customers. We therefore normalize $\lambda_{L}, N_{L}$ and $d_{L}$ at their respective unit values to simplify analysis. The U.S. Census Bureau [8] estimates the number of U.S. households in 2003 to be about 111.2 million, and given the number of broadband access lines of about 28.2 million households, currently about a quarter of the U.S. households receive some form of broadband access. We therefore vary $N_{H}$ in the range $[0.1,0.4]$. We assume that "true" broadband access ranges between 1.5 to 5 Mbps, while 'lite' broadband access is supposed to be $256 \mathrm{kbps}$. Therefore, we vary $\lambda_{H}$ in the range $[6,30]$ as compared to the normalized unit value of $\lambda_{L}$. Finally, without reliable data to serve as proxy for costs of delays for the two different classes of users, we somewhat arbitrarily vary $d_{H}$ between the values 5 and 10, keeping $d_{L}$ normalized. Figure 1 shows the change in the profit functions with $d_{H}=5$, while Figure 2 shows the change in the profit functions with $d_{H}=10$. In both the figures, the relatively flat meshed surface shows the profit $\Pi_{2}$ under Option 2, the smooth upward-sloping surface represents the profit $\Pi_{1}$ or $\Pi_{3}$ under Options 1 and 3, and the meshed upward-sloping surface represents the profit $\Pi_{4}$ under Option 4. 
As the figures indicate, option 2 (i.e. selling to both types of customers charging a single fixed fee) gives the provider highest profits when the relative magnitudes of $\left(\frac{N_{H}}{N_{L}}\right)$ and $\left(\frac{\lambda_{H}}{\lambda_{L}}\right)$ are low. Thus, introducing on-demand access does not make sense when the number of high-speed users and their relative bandwidth requirements are low. Option 1 (i.e. selling to high-type users only) make sense at intermediate values of $\left(\frac{N_{H}}{N_{L}}\right)$ and $\left(\frac{\lambda_{H}}{\lambda_{L}}\right)$, while at higher values of these ratios, introducing liquid pricing for low-type users becomes the most preferred option. Thus, in the early stages of introduction of broadband services, service providers would probably be best off by serving both types of customers with one fixed access charge per month. As broadband services become more popular, we run into an interesting situation where the monopolist provider might not want to service the low-type users (option 1 or 3), a situation that regulators might want to be aware of. To ensure that the low-type users are not left uncovered, regulators might want to subsidize the monopolist provider, which might manifest itself in the form of universal service funds (USF) that many telecom providers nowadays charge for expanding telephone services to schools and rural areas. This subsidy should be dropped as broadband penetration rates increase and access speeds get faster.

We make the observation that at current penetration rates for broadband (around 25\%) and the gradual introduction of broadband access at 3 Mbps or higher, option 2 would 
probably not be the preferred route for most service providers today except in very nascent market segments.

\section{Model 2}

In the second scenario, we assume that the low-type customers need their "normal” lowbandwidth services most of the time, and access to high-bandwidth services for $\alpha$ fraction of their time. We also assume that the provider employs two dedicated service capacities, $\mu_{1}$ and $\mu_{2}$ where $\mu_{1} \gg \mu_{2}$. In terms of notation, we have the following:

$N_{H}$ : number of high-type customers

$N_{L}:$ number of low-type customers

$\lambda_{H}$ : High-type customer's arrival rate of requests per unit of time, and that of the lowtype's for $\alpha$ fraction of the time.

$\lambda_{L}$ : Low-type customer's “normal” arrival rate of requests (packets) per unit of time

$\alpha$ : proportion of the time of the low-type customers whose arrival rate of requests is $\lambda_{H}$, so that in the other $(1-\alpha)$ proportion of the time the arrival rate is $\lambda_{L}$.

$d_{H}$ : delay cost per unit of time of High type customers and the high traffic of low-type customers

$d_{L}$ : delay cost per unit of time of the normal traffic of low-type customers, where $d_{H}>d_{L}$.

$\mu_{1}$ : service capacity of the channel for the high-type customers (channel 1), per unit of time

$\mu_{2}$ : service capacity of the channel for the low-type customers (channel 2), per unit of time, where $\mu_{1} \gg \mu_{2}$.

$F_{i}$ : fixed fee per unit of time, where $i \in\{5,6,7,8,9\}$ for selecting the two service capacities in the options 5, 6 and 8 we describe below 
$p_{j}$ : usage charge per unit of time, $j \in\{3,4\}$ in options 7 and 8 that we describe below

$v\left(\lambda_{k}\right)$ : gross value function of using the service for the two types of customers, $k \in\{H, L\}$, twice differentiable and concave

\section{Analysis}

Once again, we assume that the service provider has a matrix of strategies, depending on whether she wants to cater to both type of customers, or just to the high-type, as well as her decision to introduce on-demand services. The various possible strategies for the provider are summarized in Table 2.

Table 2: Product Strategies of the Digital Infrastructure Services

\begin{tabular}{lll}
\hline & Sell to High type only & Sell to both types \\
\hline No on-demand & Option 5 & Option 6 \\
& $\Pi_{5}=N_{H} F_{5}$ & $\Pi_{6}=N_{H} F_{6}+N_{L} F_{7}$ \\
\hline Introduce on-demand & Option 7 & Option 8 \\
& $\Pi_{7}=N_{H} p_{3} \lambda_{H}$ & $\Pi_{8}=N_{H} F_{8}+N_{L} p_{4} \alpha \lambda_{H}+N_{L} F_{9}$ \\
\hline
\end{tabular}

Option 5 refers to the decision where the provider caters just to the high-type customers for a fixed fee. Option 6 refers to the decision where the high-type customers are served through the high-bandwidth capacity $\mu_{1}$, and the low-type customers are served through the low-bandwidth capacity $\mu_{2}$. In option 7 , the provider serves the high-type customers only for a per-unit access charge. In option 8, we model the real-world scenario, where the high-type customers are served through the high-bandwidth capacity $\mu_{1}$, and the lowtype customers are served through the low-bandwidth capacity $\mu_{2}$; in addition, for an 
$\alpha$ fraction of the time, the low-type customers have a requirement for high-bandwidth services, with an arrival rate of $\lambda_{H}$, for which they pay a per-unit access charge of $p_{4}$.

The profit maximization principle of the provider in each of the above options is as follows:

Option 5:

$$
\begin{aligned}
& \max _{F_{5}} \Pi_{5}=N_{H} F_{5} \\
& \text { s.t. } \quad v\left(\lambda_{H}\right)-\frac{d_{H}}{\mu_{1}-N_{H} \lambda_{H}}-F_{5} \geq 0
\end{aligned}
$$

Option 6:

$$
\begin{gathered}
\max _{F_{6}, F_{7}} \Pi_{6}=\left(N_{H} F_{6}+N_{L} F_{7}\right) \\
\text { s.t. } \alpha\left[v\left(\lambda_{H}\right)-\frac{d_{H}}{\mu_{2}-N_{L} \lambda_{H}}\right]+(1-\alpha)\left[v\left(\lambda_{L}\right)-\frac{d_{L}}{\mu_{2}-N_{L} \lambda_{L}}\right]-F_{7} \\
\geq \alpha\left[v\left(\lambda_{H}\right)-\frac{d_{H}}{\mu_{1}-N_{L} \lambda_{H}}\right]+(1-\alpha)\left[v\left(\lambda_{L}\right)-\frac{d_{L}}{\mu_{1}-N_{L} \lambda_{L}}\right]-F_{6} \\
\alpha\left[v\left(\lambda_{H}\right)-\frac{d_{H}}{\mu_{2}-N_{L} \lambda_{H}}\right]+(1-\alpha)\left[v\left(\lambda_{L}\right)-\frac{d_{L}}{\mu_{2}-N_{L} \lambda_{L}}\right]-F_{7} \geq 0 \\
v\left(\lambda_{H}\right)-\frac{d_{H}}{\mu_{1}-N_{H} \lambda_{H}}-F_{6} \geq v\left(\lambda_{H}\right)-\frac{d_{H}}{\mu_{2}-N_{H} \lambda_{H}}-F_{7} \\
v\left(\lambda_{H}\right)-\frac{d_{H}}{\mu_{1}-N_{H} \lambda_{H}}-F_{6} \geq 0
\end{gathered}
$$

\section{Option 7:}




$$
\begin{aligned}
& \max _{p_{3}} \Pi_{7}=N_{H} p_{3} \lambda_{H} \\
& \text { s.t. } \quad v\left(\lambda_{H}\right)-\frac{d_{H}}{\mu_{1}-N_{H} \lambda_{H}}-p_{3} \lambda_{H} \geq 0
\end{aligned}
$$

\section{Option 8:}

$$
\begin{aligned}
& \max _{F_{8}, p_{4}, F_{9}} \Pi_{8}=N_{H} F_{8}+N_{L} p_{4} \alpha \lambda_{H}+N_{L} F_{9} \\
& \text { s.t. } \\
& \begin{array}{l}
\alpha\left[v\left(\lambda_{H}\right)-\frac{d_{H}}{\mu_{1}-N_{L} \lambda_{H}}-p_{4} \lambda_{H}\right]+(1-\alpha)\left[v\left(\lambda_{L}\right)-\frac{d_{L}}{\mu_{2}-N_{L} \lambda_{L}}\right]-F_{9} \\
\geq \alpha\left[v\left(\lambda_{H}\right)-\frac{d_{H}}{\mu_{1}-N_{L} \lambda_{H}}-p_{4} \lambda_{H}\right]+(1-\alpha)\left[v\left(\lambda_{L}\right)-\frac{d_{L}}{\mu_{1}-N_{L} \lambda_{L}}-p_{4} \lambda_{L}\right]
\end{array} \\
& \alpha\left[v\left(\lambda_{H}\right)-\frac{d_{H}}{\mu_{1}-N_{L} \lambda_{H}}-p_{4} \lambda_{H}\right]+(1-\alpha)\left[v\left(\lambda_{L}\right)-\frac{d_{L}}{\mu_{2}-N_{L} \lambda_{L}}\right]-F_{9} \\
& \geq \alpha\left[v\left(\lambda_{H}\right)-\frac{d_{H}}{\mu_{1}-N_{L} \lambda_{H}}\right]+(1-\alpha)\left[v\left(\lambda_{L}\right)-\frac{d_{L}}{\mu_{1}-N_{L} \lambda_{L}}\right]-F_{8} \\
& \alpha\left[v\left(\lambda_{H}\right)-\frac{d_{H}}{\mu_{1}-N_{L} \lambda_{H}}-p_{4} \lambda_{H}\right]+(1-\alpha)\left[v\left(\lambda_{L}\right)-\frac{d_{L}}{\mu_{2}-N_{L} \lambda_{L}}\right]-F_{9} \\
& \geq \alpha\left[v\left(\lambda_{H}\right)-\frac{d_{H}}{\mu_{2}-N_{L} \lambda_{H}}\right]+(1-\alpha)\left[v\left(\lambda_{L}\right)-\frac{d_{L}}{\mu_{2}-N_{L} \lambda_{L}}\right]-F_{9} \\
& \alpha\left[v\left(\lambda_{H}\right)-\frac{d_{H}}{\mu_{1}-N_{L} \lambda_{H}}-p_{4} \lambda_{H}\right]+(1-\alpha)\left[v\left(\lambda_{L}\right)-\frac{d_{L}}{\mu_{2}-N_{L} \lambda_{L}}\right]-F_{9} \geq 0 \\
& v\left(\lambda_{H}\right)-\frac{d_{H}}{\mu_{1}-N_{H} \lambda_{H}}-F_{8} \geq v\left(\lambda_{H}\right)-\frac{d_{H}}{\mu_{1}-N_{H} \lambda_{H}}-p_{4} \lambda_{H} \\
& v\left(\lambda_{H}\right)-\frac{d_{H}}{\mu_{1}-N_{H} \lambda_{H}}-F_{8} \geq v\left(\lambda_{H}\right)-\frac{d_{H}}{\mu_{2}-N_{H} \lambda_{H}}-F_{9} \\
& v\left(\lambda_{H}\right)-\frac{d_{H}}{\mu_{1}-N_{H} \lambda_{H}}-F_{8} \geq 0
\end{aligned}
$$


The details of the derivations are provided in the Mathematical Appendix. For options 5 and 7, we get,

$$
\Pi_{5}=\Pi_{7}=N_{H}\left(v\left(\lambda_{H}\right)-\frac{d_{H}}{\mu_{1}-N_{H} \lambda_{H}}\right)
$$

Equations (A1) and (A2) in the mathematical appendix essentially indicate that the service provider extracts all the rent in options 5 and 7, and therefore the consumers have a zero surplus.

In option 6, constraints (19) and (20) are the incentive compatibility and participation constraints of the low-type customer, (21) and (22) are the incentive compatibility and participation constraints of the high-type customer. For tractability purposes, we assume that most of the users are the low bandwidth users, so that not only $N_{L}>N_{H}$ but also $N_{L} \lambda_{L}>N_{H} \lambda_{H}$, i.e. in terms of total bandwidth usage, the low-type users cumulatively consume more of the bandwidth than the high-type users. We further assume that $F_{6}>F_{7}$, i.e. the fixed charge for the low-type users is lower than that of the high-type users, something which is readily observable and expected in real life. Now, with $\mu_{1}>\mu_{2}$, one can verify that (19) is a strict inequality, and therefore the incentive compatibility constraint of the low-type user is not binding.

From the analysis detailed in the mathematical appendix, we see that the profit in option 6 is given by 


$$
\begin{aligned}
\Pi_{6}= & N_{H}\left\{\begin{array}{l}
\alpha\left[v\left(\lambda_{H}\right)-\frac{d_{H}}{\mu_{2}-N_{L} \lambda_{H}}\right]+(1-\alpha)\left[v\left(\lambda_{L}\right)-\frac{d_{L}}{\mu_{2}-N_{L} \lambda_{L}}\right] \\
+d_{H}\left(\frac{1}{\mu_{2}-N_{H} \lambda_{H}}-\frac{1}{\mu_{1}-N_{H} \lambda_{H}}\right)
\end{array}\right] . \\
& +N_{L}\left\{\alpha\left[v\left(\lambda_{H}\right)-\frac{d_{H}}{\mu_{2}-N_{L} \lambda_{H}}\right]+(1-\alpha)\left[v\left(\lambda_{L}\right)-\frac{d_{L}}{\mu_{2}-N_{L} \lambda_{L}}\right]\right\}
\end{aligned}
$$

The surplus of the low-type consumer is zero, while that of the high-type is given by the expression

$$
(1-\alpha)\left[v\left(\lambda_{H}\right)+v\left(\lambda_{L}\right)-\frac{d_{L}}{\mu_{2}-N_{L} \lambda_{L}}\right]+d_{H}\left(\frac{\alpha}{\mu_{2}-N_{L} \lambda_{H}}+\frac{1}{\mu_{2}-N_{H} \lambda_{H}}-\frac{2}{\mu_{1}-N_{H} \lambda_{H}}\right) .
$$

In option 8 , for tractability purposes, we make a simplifying assumption that the two types of users have the entire dedicated channel bandwidths to themselves when they are using a particular channel. Further, as before, we assume that most of the users are the low bandwidth users, so that not only $N_{L}>N_{H}$ but also $N_{L} \lambda_{L}>N_{H} \lambda_{H}$, i.e. in terms of total bandwidth usage, the low-type users cumulatively consume more of the bandwidth than the high-type users.

The derivations in the mathematical appendix shows that the profits in this option is given by

$$
\begin{aligned}
& \Pi_{8}=\left\{\begin{array}{l}
\frac{1}{1+\alpha}\left[\alpha\left\{v\left(\lambda_{H}\right)-\frac{d_{H}}{\mu_{2}-N_{H} \lambda_{H}}\right\}+(1-\alpha)\left\{v\left(\lambda_{L}\right)-\frac{d_{L}}{\mu_{2}-N_{L} \lambda_{L}}\right\}\right] \\
+d_{H}\left(\frac{1}{\mu_{2}-N_{H} \lambda_{H}}-\frac{1}{\mu_{1}-N_{H} \lambda_{H}}\right)
\end{array}\right\}\left(N_{H}+\alpha N_{L}\right) \\
& +N_{L}\left(\frac{1}{1+\alpha}\left[\alpha\left\{v\left(\lambda_{H}\right)-\frac{d_{H}}{\mu_{2}-N_{H} \lambda_{H}}\right\}+(1-\alpha)\left\{v\left(\lambda_{L}\right)-\frac{d_{L}}{\mu_{2}-N_{L} \lambda_{L}}\right\}\right]\right)
\end{aligned}
$$


Equation (A8) indicates that the per-unit charge for the high-channel is such that it just dissuades the high-type of user from choosing per-unit charges. Equation (A9) shows that the fixed price differential between the high-channel and the low-channel, which is the differential that high-type user pays for his service (as compared to the low type), is equal to the difference in the delay cost of the high-type of user between using the low-channel and him using the high-channel. In other words, the premium over the price of the lowchannel that the high type-user is willing to pay for the high-channel is equal to the extra delay cost he incurs in using the low-channel. Thus, equation (A10) gives us the maximum fixed price that the provider can extract for the low-channel, which can then be inserted in equation (A9) to get the price premium that the provider can charge for the high-channel. This price premium provides the basis for the provider to decide on the maximum per-unit charge that he can command from the low-type users for the highchannel in equation (A8). Once again the consumer surplus of the low-type user is zero, while that of the high type is given by the following expression:

$v\left(\lambda_{H}\right)-\left\{\frac{1}{1+\alpha}\left[\alpha\left\{v\left(\lambda_{H}\right)-\frac{d_{H}}{\mu_{2}-N_{H} \lambda_{H}}\right\}+(1-\alpha)\left\{v\left(\lambda_{L}\right)-\frac{d_{L}}{\mu_{2}-N_{L} \lambda_{L}}\right\}\right]+\left(\frac{d_{H}}{\mu_{2}-N_{H} \lambda_{H}}-\frac{d_{H}}{\mu_{1}-N_{H} \lambda_{H}}\right)\right\}$

As before, we plot the profit functions in the various options available to the service provider. The specific values of the parameters are kept the same as before, except that in order to keep the relative magnitudes of the available bandwidths as per our assumption $\mu_{1} \gg \mu_{2}$, we stipulate that $\mu_{1}=500$ and $\mu_{2}=50$. We further assume that the fraction of time that the low-type users feel the need for high bandwidth services, $\alpha=0.1$. Figures 3 and 4 show the change in the profit functions under the various options as the ratios 
$\left(\frac{N_{H}}{N_{L}}\right)$ and $\left(\frac{\lambda_{H}}{\lambda_{L}}\right)$ change. In both the figures, the relatively flat meshed surface shows the profit $\Pi_{6}$ under Option 6, the smooth surface represents the profit $\Pi_{5}$ or $\Pi_{7}$ under Options 5 and 7, and the meshed upward-sloping surface represents the profit $\Pi_{8}$ under Option 8.

\section{--- Insert Figures 3 and 4 About Here ---}

It is interesting to note that within the range of variation of the ratios of the parameters $\left(\frac{N_{H}}{N_{L}}\right)$ and $\left(\frac{\lambda_{H}}{\lambda_{L}}\right)$, option 6 (selling to both types of users with different fixed prices) never yields the highest profits for the monopolist service provider. Thus, at least under the conditions of this model, a service like AOL Broadband to coexist with the regular dialup AOL service is counter-productive. For relatively lower values of the two ratios, the monopolist will service only the high-type of users, and at higher values of the two ratios, serving both types of customers with the option of liquid pricing on demand for the low-type of users the becomes the most attractive option.

As in Model 1, the results of this stylized model indicate that the monopolist provider might have to be subsidized or regulated in some fashion so that she provides highbandwidth services at the initial stages, when the number of early adopters is low. Once the service gets more established among the consumers, and the service provider begins 
to provide higher access rates, the liquid pricing option becomes the most lucrative, and the subsidy (or regulation) should then be removed.

\section{Conclusion}

This paper looks at the effect of introducing on-demand access with liquid pricing for digital infrastructure services, like broadband Internet, wireless communication, etc. The twin effects of infrastructure overcapacity and relatively low penetration of highbandwidth services has prompted the introduction of intermediate level services to the general population, with the hope that in the long term, these consumers would gradually upgrade their services. This paper shows that introducing liquid pricing for on-demand access to broadband services can increase the profits of the service provider. Introduction of such a service would have practically zero costs, especially in an environment of infrastructure overcapacity, but can significantly increase revenue and profits. The results also indicates that the monopolist might sometimes prefer to service only the high-type of user, which might therefore prompt regulators to either force her to service both types of users, or otherwise subsidize her in some other fashion (assuming of course that universal access to broadband services is the desired goal of the society).

As mentioned in the introduction, bandwidth service providers have an opportunity of revenue-sharing with providers of broadband content. Organizations like Movielink (downloadable feature films), Real Networks (broadband entertainment), and the rapidly growing digital music industry would greatly benefit from increased adoption of broadband services by users who might value the traditional Internet access very 
differently from the new online content. A similar phenomenon is gradually taking shape in the mobile telecommunications industry.

An interesting extension of this research would be to model the effect of the availability and adoption of broadband content. In South Korea, which has the highest per-capita penetration of broadband services in the world, broadband service providers are gradually moving into providing broadband content in order to drive revenues [12]. One could therefore model this environment as a "content provider-access provider-consumer" supply chain and analyze whether these content providers should subsidize broadband access in order to grow the market, as opposed to intervention by a regulatory body that we indicate in our model. 


\section{References}

1. Between a rock and a hard place, The Economist, Vol. October, October 9 (2003), Special Supplement.

2. New figures show broadband deployment accelerating in the US, Federal Communications Commission, September (2004).

3. Bandyopadhyay, S., and Cheng, H.K., Broadband pricing, universal access and implications for public policy. 2005, Warrington College of Business, University of Florida: 23.

4. Bhargava, H.K., and Sun, D. Quality-contingent pricing for broadband services, in Proceedings of HICSS-38, Hawaii, 2005.

5. Bhargava, H.K., and Sundaresan, S. Contingency pricing for information goods and services under industrywide performance standard. Journal of Management Information Systems, 20, 2 (2003) 113-136.

6. Dobson, G., and Kalish, S. Positioning and pricing a product line. Marketing Science, 7, 2 (1988) 107-125.

7. Edell, R.J., and Varaiya, P.P. Providing Internet Access: What we learn from the INDEX trial, INDEX Project Report \#99-010W, University of California at Berkeley, (1999).

8. Fields, J. American families and living arrangements, U.S. Census Bureau, November (2004).

9. Gardner, W.D. Broadband 'Redlining' Issue Raised In Fiber Deployment, (2005). http://www.techweb.com/wire/networking/60400165

10. Gomes, L. Despite Opposition, Might the Web Need New Government Jolt? The Wall Street Journal, February 14 2005, B1.

11. Haruvy, E., and Prasad, A. Optimal product strategies in the presence of network externalities. Information Economics and Policy, 10 (1998) 489-499.

12. Hong, M.Y., and Kim, S. Broadband's future: lessons from South Korea. The McKinsey Quarterly, 4, (2004) 9-11.

13. Lilien, G.L.; Kotler, P.; and Moorthy, K. Marketing models. Prentice Hall, 1992.

14. Mackie-Mason, J.K., and Varian, H.R. Pricing the Internet, in Proceedings of Public Access to the Internet, JFK School of Government, 1993.

15. Mackie-Mason, J.K., and Varian, H.R. Some economics of the internet. ed. W. Sichel, University of Michigan Press, Ann Arbor, MI, 1996. 
16. Mendelson, H. Pricing computer services: queueing effects. Communications of the ACM, 28, 3 (1985) 312-321.

17. Moorthy, K.S. Market segmentation, self-selection and product line design. Marketing Science, 3, 4 (1984) 288-307.

18. Mossberg, W. Verizon devices use high-speed network for voice, web, e-mail, The Wall Street Journal, December 16 2004, C1.

19. Mussa, M., and Rosen, S. Monopoly and product quality. Journal of Economic Theory, 18, 2 (1978) 301-317.

20. Odlyzko, A. Paris Metro pricing for the internet, in Proceedings of ACM Conference on Electronic Commerce, 1998140-147.

21. Squeo, A.M. In Tiny Towns, New Call Options Shake Up an Old Phone System, The Wall Street Journal, February 22 2005, A1.

22. Tirole, J. The theory of industrial organization. Prentice-Hall, 1996. 


\section{Mathematical appendix}

Options 5 and 7:

Analytically, options 5 and 7 are relatively simple, with the only participation constraints (17) and (23) being binding. Therefore,

$$
F_{5}=v\left(\lambda_{H}\right)-\frac{d_{H}}{\mu_{1}-N_{H} \lambda_{H}}
$$

and

$$
p_{3}=\frac{v\left(\lambda_{H}\right)}{\lambda_{H}}-\frac{d_{H} / \lambda_{H}}{\mu_{1}-N_{H} \lambda_{H}}
$$

Option 6:

We re-write the right-hand side of inequality (21) as

$$
\alpha\left[v\left(\lambda_{H}\right)-\frac{d_{H}}{\mu_{2}-N_{H} \lambda_{H}}-F_{7}\right]+(1-\alpha)\left[v\left(\lambda_{H}\right)-\frac{d_{H}}{\mu_{2}-N_{H} \lambda_{H}}-F_{7}\right]
$$

and compare it with the left-hand side of the inequality (20). Since $F_{6}>F_{7}, \mu_{1}>\mu_{2}$ and $N_{L} \lambda_{L}>N_{H} \lambda_{H}$, one can verify that the former quantity is greater than the latter. But since the left-hand side of (21) is the net utility of the high-type user, and the left-hand side of (20) is the net utility of the low-type user, which by the participation constraint of the low-type user is non-negative, it follows therefore that the net utility of the high-type user is strictly positive, or in other words, the participation constraint of the high-type user (22) is non-binding. Thus, the only participation constraint that the provider is concerned with is that of the low-type, and the provider will increase the fixed cost $F_{7}$, so that it is just worthwhile for the low-type to participate (thus the low-type has zero surplus). In other words, inequality (20) is binding. The high-type will therefore extract a strictly positive surplus, and the provider will do such that it is just sufficient for the high-type to 
not choose a contract that encourages him to choose the low-channel for a fixed fee. In other words, inequality (21) will be binding.

We therefore solve for $F_{6}$ and $F_{7}$ from these two binding constraints,

$$
\begin{aligned}
F_{6}= & \alpha\left[v\left(\lambda_{H}\right)-\frac{d_{H}}{\mu_{2}-N_{L} \lambda_{H}}\right]+(1-\alpha)\left[v\left(\lambda_{L}\right)-\frac{d_{L}}{\mu_{2}-N_{L} \lambda_{L}}\right] \\
& +d_{H}\left(\frac{1}{\mu_{2}-N_{H} \lambda_{H}}-\frac{1}{\mu_{1}-N_{H} \lambda_{H}}\right) \\
F_{7}= & \alpha\left[v\left(\lambda_{H}\right)-\frac{d_{H}}{\mu_{2}-N_{L} \lambda_{H}}\right]+(1-\alpha)\left[v\left(\lambda_{L}\right)-\frac{d_{L}}{\mu_{2}-N_{L} \lambda_{L}}\right]
\end{aligned}
$$

Option 8:

Inequality (24) is the incentive compatibility of the low-type user, whereby his payoff from using the high-channel $\alpha$ fraction of the time (with liquid pricing) and using the low-channel the rest of the time (with its associated fixed cost) is higher than using the on-demand channel at all times. Inequality (25) is another incentive compatibility constraint of the low-type so that he prefers his current setup as compared to using the high-channel all the time with the fixed price of $F_{8}$. Inequality (26) is the incentive compatibility constraint of the low-type so that he prefers his current setup as compared to using the low-channel at all times for the fixed fee of $F_{9}$. Similarly, inequalities (28) and (29) are the incentive compatibility constraints of the high-type user, so that he prefers using the high-channel for a fixed fee rather than paying per-unit charges, and further, he prefers using the high-channel at all times than the low-channel at all times, respectively. Inequality (27) is the participation constraint of the low-type user, and (30) is the participation constraint of the high-type user. 
Multiplying both sides of (28) by $\alpha$, and both sides of (29) by $(1-\alpha)$, and adding them up, gives us the following inequality

$$
\begin{aligned}
& v\left(\lambda_{H}\right)-\frac{d_{H}}{\mu_{1}-N_{H} \lambda_{H}}-F_{8} \geq \\
& \quad \alpha\left[v\left(\lambda_{H}\right)-\frac{d_{H}}{\mu_{1}-N_{H} \lambda_{H}}-p_{4} \lambda_{H}\right]+(1-\alpha)\left[v\left(\lambda_{H}\right)-\frac{d_{H}}{\mu_{2}-N_{H} \lambda_{H}}\right]-(1-\alpha) F_{9}
\end{aligned}
$$

Since $0<\alpha<1,(1-\alpha) F_{9}<F_{9}$, and therefore we can rewrite inequality (A6) as

$$
\begin{aligned}
& v\left(\lambda_{H}\right)-\frac{d_{H}}{\mu_{1}-N_{H} \lambda_{H}}-F_{8} \\
& >\alpha\left[v\left(\lambda_{H}\right)-\frac{d_{H}}{\mu_{1}-N_{H} \lambda_{H}}-p_{4} \lambda_{H}\right]+(1-\alpha)\left[v\left(\lambda_{H}\right)-\frac{d_{H}}{\mu_{2}-N_{H} \lambda_{H}}\right]-F_{9}
\end{aligned}
$$

We then compare the left side of inequality (27) with the right side of inequality (A6a), and the latter is greater than the former if the following inequality (after simplification) holds true:

$$
\begin{aligned}
& (1-\alpha)\left[\left\{v\left(\lambda_{H}\right)-v\left(\lambda_{L}\right)\right\}-\left\{\frac{d_{H}}{\mu_{2}-N_{H} \lambda_{H}}-\frac{d_{L}}{\mu_{2}-N_{L} \lambda_{L}}\right\}\right] \\
& +\alpha\left[\frac{d_{H}}{\mu_{1}-N_{L} \lambda_{H}}-\frac{d_{H}}{\mu_{1}-N_{H} \lambda_{H}}\right]>0
\end{aligned}
$$

Since $v\left(\lambda_{H}\right)>v\left(\lambda_{L}\right), d_{H}>d_{L}, N_{L}>N_{H}$ and $N_{L} \lambda_{L}>N_{H} \lambda_{H}$, it is easy to verify that the above inequality holds. This means that the left hand side of the inequality (A6) is strictly 
greater than the left side of the inequality (27). But the left side of inequality (27) is the participation constraint of the low-type user, which is a non-negative quantity. Since the left side of inequality (A6) is the rent of the high-type of user, it means that this value is strictly positive, and therefore inequality (30) - the participation constraint of the hightype - is not binding. Thus, the only participation constraint that the provider should consider is that of the low-type, and the provider will increase the fixed cost $F_{9}$ and the per-unit charges $p_{4}$, so that it is just worthwhile for the low-type to participate. In other words, inequality (27) is binding (ensuring zero surplus for the low-type customer). The high-type user will extract a strictly positive surplus, and the provider will do such that it is just sufficient for the high-type to not choose a contract that either encourages him to choose the low-channel for a fixed fee or the high-channel using per-unit charges. In other words, inequalities (28) and (29) will be binding. Thus, we get three equations, to solve for the three unknowns $\left(F_{8}, F_{9}, p_{4}\right)$.

Solving, we get

$$
\begin{gathered}
p_{4} \lambda_{H}=F_{8} \\
F_{8}-F_{9}=\frac{d_{H}}{\mu_{2}-N_{H} \lambda_{H}}-\frac{d_{H}}{\mu_{1}-N_{H} \lambda_{H}} \\
F_{9}=\frac{1}{1+\alpha}\left[\alpha\left\{v\left(\lambda_{H}\right)-\frac{d_{H}}{\mu_{2}-N_{H} \lambda_{H}}\right\}+(1-\alpha)\left\{v\left(\lambda_{L}\right)-\frac{d_{L}}{\mu_{2}-N_{L} \lambda_{L}}\right\}\right]
\end{gathered}
$$




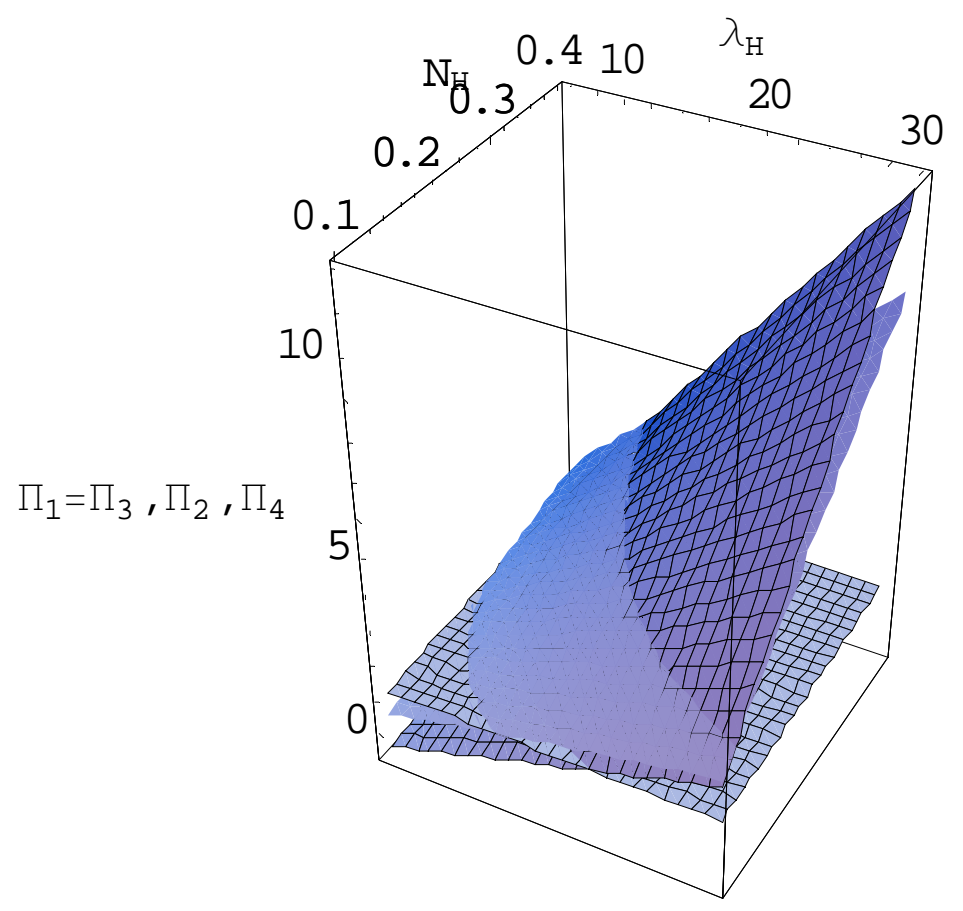

Figure 1: Relative magnitudes of the profits under the various scenarios in Model 1 by changing the ratios $\left(\frac{N_{H}}{N_{L}}\right)$ and $\left(\frac{\lambda_{H}}{\lambda_{L}}\right)$ and $d_{H}=5$ 


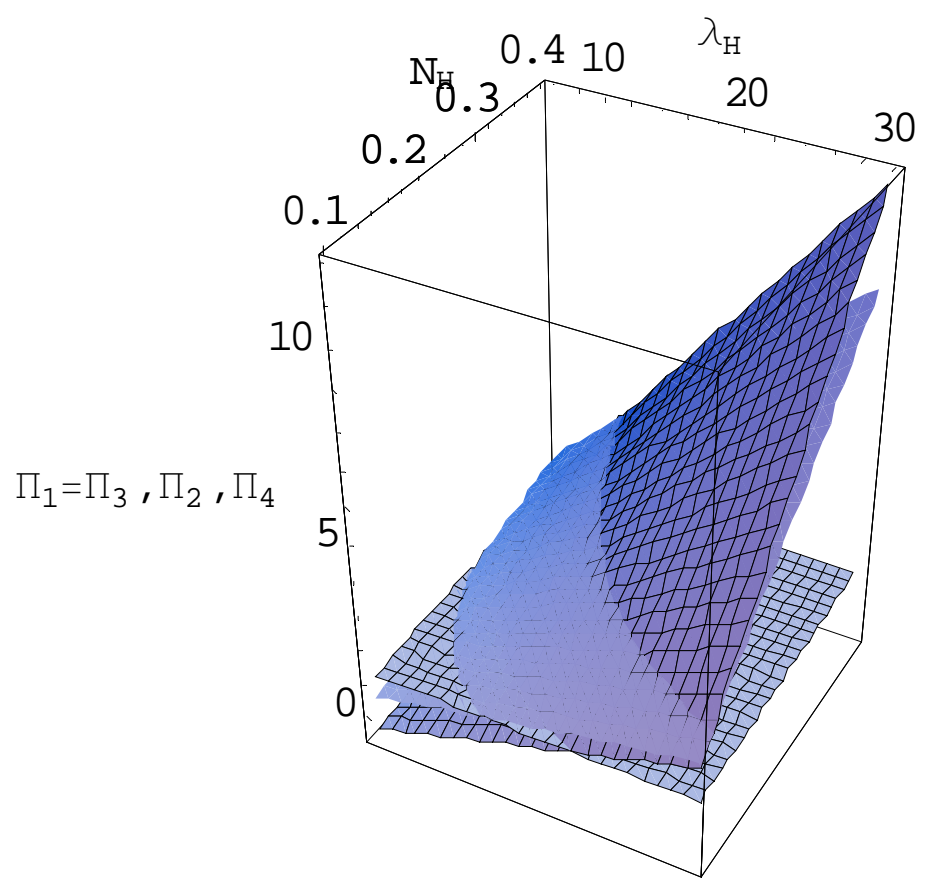

Figure 2: Relative magnitudes of the profits under the various scenarios in Model 1 by changing the ratios $\left(\frac{N_{H}}{N_{L}}\right)$ and $\left(\frac{\lambda_{H}}{\lambda_{L}}\right)$ and $d_{H}=10$ 


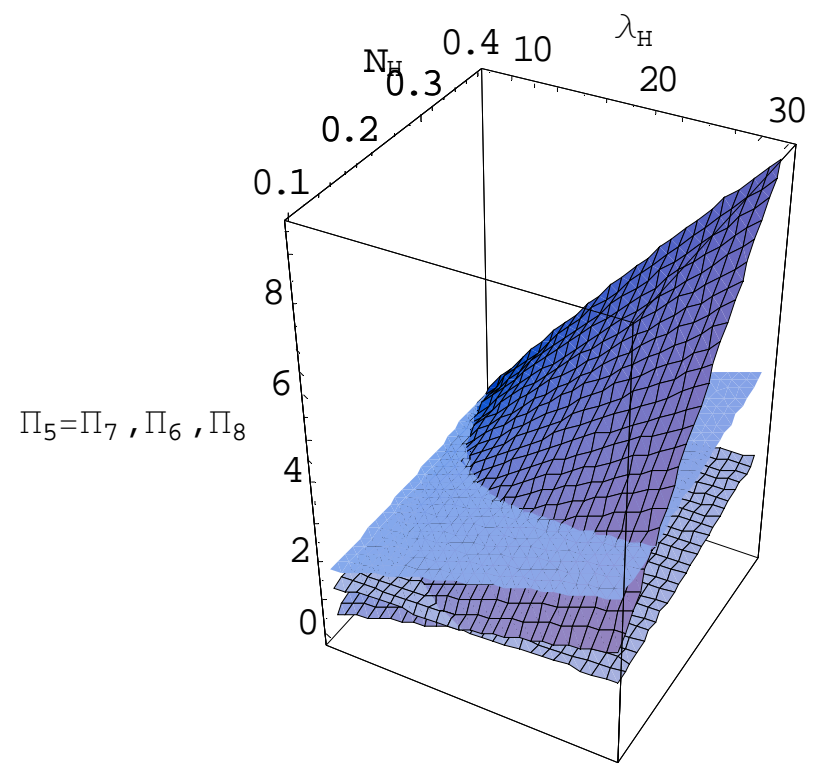

Figure 3: Relative magnitudes of the profits under the various scenarios in Model 2 by changing the ratios $\left(\frac{N_{H}}{N_{L}}\right)$ and $\left(\frac{\lambda_{H}}{\lambda_{L}}\right)$ and $d_{H}=5$ 


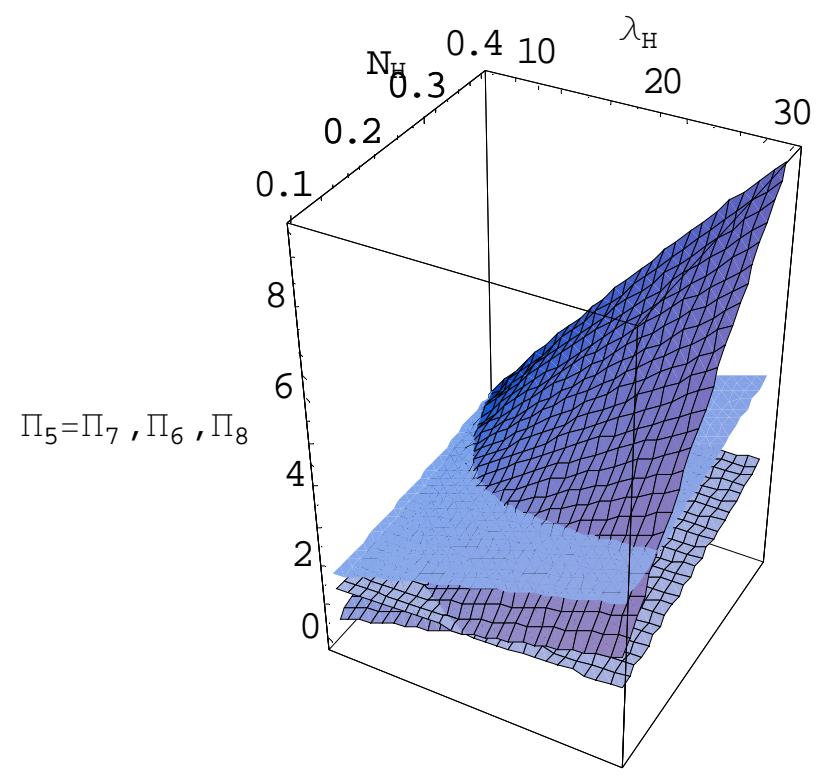

Figure 4: Relative magnitudes of the profits under the various scenarios in Model 2 by changing the ratios $\left(\frac{N_{H}}{N_{L}}\right)$ and $\left(\frac{\lambda_{H}}{\lambda_{L}}\right)$ and $d_{H}=10$ 